\title{
Appropriate And Inappropriate Fresh Frozen Plasma (Ffp) Use In Coagulation Disorders
}

\author{
M. A ${ }^{-S a m e e r}{ }^{1}$, S. A. Deshpande ${ }^{2}$, Vikas Dagar ${ }^{3}$, Santosh Pawar $^{3}$, \\ D. P. Meshram ${ }^{4}$ \\ ${ }^{1}$ Associate professor,department of pathology, ,Dr. Shankarrao chavan government medical college \\ nanded,India \\ ${ }^{2}$ Professor and head, department of pathology, ,Dr. Shankarrao chavan government medical college \\ nanded,India \\ ${ }^{3}$ P.G. Resident,department of pathology, ,Dr. Shankarrao chavan government medical college nanded,India \\ ${ }^{4}$ Assisitant professor,department of pathology, ,Dr. Shankarrao chavan government medical college \\ nanded,India
}

\begin{abstract}
:
AIMS:The aim of this study was to evaluate the usage of fresh frozen plasma (FFP) according to indications and to reduce inappropriate usage.

Method: A two year retrospective study was conducted in Dr.S.C.G.M.C.and hospital blood bank . Based on the guidelines published by college of American pathologist,national health and medical research council and Australian society for blood transfusion FFP usage were categorized into appropriate and inappropriate.Pre and post transfusion INR/PT were recorded and the effect of FFP were studied in patients who received FFP

Results:During two year study 1079 unit of FFP were used for 267 patients.out of 267 patients only $125(46.81 \%)$ request were appropriate and $142(53.19 \%)$ were inappropriate requests. Pregnant female with active labour suffering from severe anaemia with shock was the commonest reason for inappropriate FFP use.Out of 125 appropriate request 100 patients were compared by evaluating Pre and post transfusion PT/INR by using fully automated coagulometer.

Conclusion: Inappropriate use of FFP not only increase the treatment costs, but also causes loss of productive power and exposes the patient to the unnecessary side effects of transfusion.inappropriate FFP transfusion should be prevented by means of educaton and awareness programme by establishing the hospital transfusion guidelines
\end{abstract}

Keywords: Appropriate, inappropriate, fresh frozen plasma (FFP,

\section{Introduction}

1.1 There is shortage of blood and blood component in most of the developing countries .

Appropriate use of blood components is required to ensure their availability for needy patients as well as to avoid the unnecessary risk of transfusion transmitted diseases .

1.2 Fresh frozen plasma (FFP) is a widely used blood component.If plasma unit is isolated from the unit of whole blood and frozen with in eight hours from donation the unit is termed as FFP.

1.3 FFP is used for coagulation disorders ,treatment of bleeding of prophylaxis of bleeding ,DIC,dilutional coagulopathy due to massive blood transfusion, in infants with secondary immunodeficiency, antithrombin deficiency and open heart surgery.

\section{Material And Method}

2.1 A two year retrospective study was conducted in Dr shankarao chavan government medical college and hospital blood bank, nanded,Maharashtra during the period January 2012 to december 2013.

2.2 The patients in whom FFP was requested and transfused were included in this study.In order to study the effect of FFP transfusion on international normalized ratio(INR) Patients who received FFP along with other supplements such as whole blood and packed red cell concentrate were excluded from this study.

2.3 Data of age ,gender of the patient provisional clinical diagnosis, indication for FFP ,department of the requesting clinician date of transfusion, number of unit transfused and coagulation profile of the patient collected.

2.4 FFP was melted at 37 degree celciusin 15 to 20 minules .Each unit of FFP was $175 \mathrm{ml}$ to $250 \mathrm{ml}$ in volume 
2.5 The guidelines published by CAP, National health and medical research council and Australian society for blood transfusion (ASBT) were used as standards as shown in table 1 and table 2.

2.6 If FFP infusion was according to the table 1 and table 2 it was categorised as appropriate transfusion otherwise as inappropriate transfusion.

2.7 The PT(prothrombin time ) was assayed and INR calculated on automated coagulometer (STA COMPACT). The pre and post transfusion PT/INR ratio were calculated in order to study the effect of FFP.The post transfusion PT/INR was done with in six hour of completion of transfusion.

\section{Results:}

3.1 A total of 1079 units of FFP were issued for 267 patients .The number of FFP infused infused per transfusion ranged from 1 to 8 with a median of 6 and average of 4.04 .

3.2 There were 115 male and 152 female with mean age of 25 year (range 1 day to $70 \mathrm{yr}$ ). FFP was most commonly transfused in the patient age group of 20 to 27 years

3.3 Most number of FFP units requested were from obstetrics and gyanaecology department followed by by medicine department as shown in table 3 .The most common indication was ANC with DIC with HELP with shock followed by liver disease .FFP was transfused to 267 patient out of which 125 were appropriate and 142 was inappropriate transfusion as per published guidelines.Highest appropriate request were from obstetrics and gynaecology department followed by medicine department Highest inappropriate request were from gynaecology department too .

3.4 Out of the 267 number of patients 201 patients received only FFP along and 66 patients received FFP with other supplements such as whole blood ,packed red cell volume, platelets e.t.c .In order to study the effect of FFP transfusion on INR,Patient who received other supplements were excluded.

3.5 Pre and post transfusion PT/INR were compared.The post transfusion PT/INR was done within six hour of transfusion.,Pre and post transfusion INR were compared in 100 patients receiving 380 transfusion of FFP.The median pretransfusion INR of these patients was 2.1(range 1.5-10.0), and the post transfusion median was 1.8(range1.3-5.2).The number of random FFP units (median volume $325 \mathrm{ml}$ ) infused per transfusion ranged from 1 to 6 with median of 2 and an average of 3.8

3.6 The effect of FFP was measured by the difference between the pre tansfusion results and the first recorded post transfusion INR.THE improvement of INR was( 0.75$)$ noted after transfusion.

3.7 None of the patients experienced any complication during the transfusions.

\section{Discussion}

4.1 Blood transfusion has been in use since many year. FFP is a frequently used blood product and evaluation of FFP usage is very important . Inspite of clear guideline regarding the use of FFP, many study has shown a high incidence of inappropriate use of FFP. ${ }^{[1]}$. Inappropriate use of FFP has a significant impact upon the patient's as well as the hospital staff in the form of healthcare cost, wastage of resources, depriving more needy patient patient and transmission of infection with unnecessary allergic reaction leading to mortality and morbidity in patients. The blood bank has to evaluate the existing trend of utility of FFP to prevent misuse which may lead to shortage and thus deny this blood product to someone in life threatening situation. In the present study, after evaluation of all the requisition form 53.19\% did not fulfill that constitute appropriate FFP usage.

4.2 FFP transfusion is appropriate in bleeding patients; patients undergoing invasive procedures with coagulopathy resulting from DIC, massive blood transfusion or liver failure and plasma exchange for thrombotic thrombocytopenic purpura [2]. In the present study the appropriate usage of FFP was $46.81 \%$ which is more than study conducted by Bhat et al.[3] which shows $32.6 \%$ appropriate usage and matches to study done by kulkarni et al [1] which shows $48 \%$ appropriate usage of FFP.

\section{Figures And Tables}

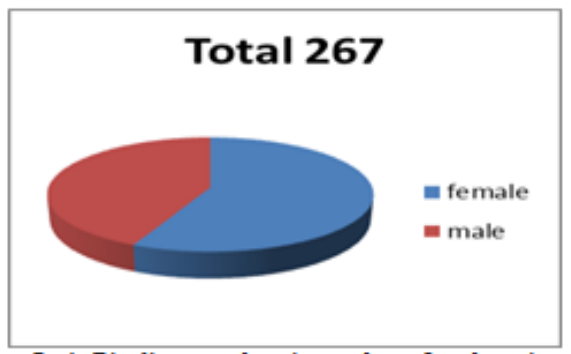

fig.1: Pie diagram showing male to female ratio. 


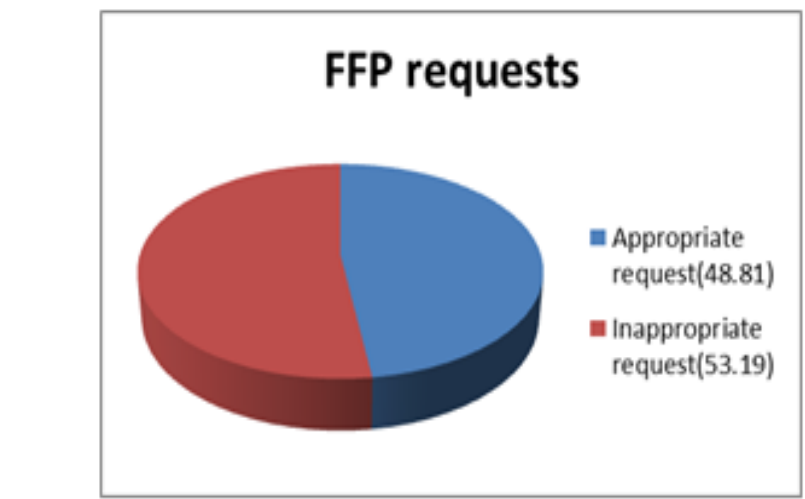

Fig 2.Pie diagram showing appropriate and inappropriate FFP requests.

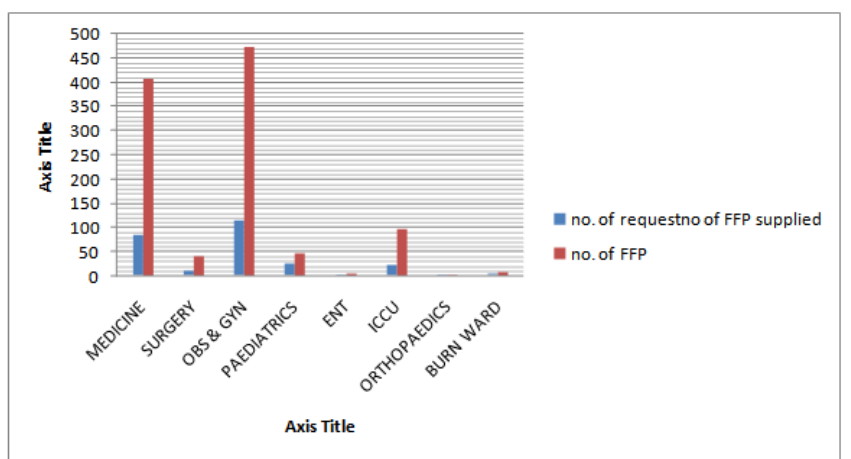

Fig 3.Departmental distribution of FFP request and number of FFP issued to respective departments.

TABLE 1: FFP Transfusiuon guidelines, college of American pathologist, 1994 [4]

1. History or clinical course suggestive of a coagulopathy due to a congenital ar acquired deficiency of coagulation factors with active bleeding or other invasive procedures. This must be documented by at least one of the following:

a.Prothrombin time (PT) greater than 1.5 times mid point of normal range.

b.Activated partial thromboplastin time (APTT) greater than 1.5 times the top of normal.

c.Coagulation assay of less than $25 \%$ activity.

2. massive blood transfusion:replacement of more than one blood unit within several hour's with evidence of a coagulation deficiency as above with continued bleeding.

3. Reversal of warfarin effect:If immediate haemostasis is required to stop active bleeding or prior to emergency surgery or an invasive procedure ( PT $>18$ seconds or INR $>1.6$ )

4. Prophylactically for surgery of invasive procedures in cases of documented congenital or acquired coagulation factor deficiency.

5. Deficiency of antithrombin,heparin cofactor, protein c or protein s.

6. Plasma exchange for thrombotic thrombocytopenic purpura of haemolytic uraemic symdrome.

TABLE 2: National health and medical research council and the Australian society for blood transfusion guidelines for transfusing fresh frozen plasma 2002. Appropriate if any of the following applicable .likely to be inappropriate if none applicable [5]

1. INR or APTT high and liver disease before major surgery or invasive procedure

2. INR or APTT high and liver failure

3. INR or APTT high and acute disseminated intravascular coagulation

4. INR or APTT high and excessive bleeding

5. INR or APTT high before an invasive procedure

6. INR or APTT high before, during or major surgery

7. INR high and warfarin effect present and massive blood loss or emergency surgery

8. correction of single factor deficiency when a specific factor was not available

9. Treatment of thrombotic thrombocytopenic purpura 
TABLE 3:Distribution of FFP request according to different departments

\begin{tabular}{|c|c|c|c|c|c|}
\hline $\begin{array}{l}\mathrm{Sr} \\
\text { no }\end{array}$ & Department & No. of request & Unit of FFP supplied & Appropriate request & $\begin{array}{l}\text { Inappropriate } \\
\text { request }\end{array}$ \\
\hline 1 & Medicine & 86 & 409 & 42 & 44 \\
\hline 2 & Surgery & 11 & 42 & 5 & 6 \\
\hline 3 & Obstetrics \& Gynaecology(OBS\& GYN) & 115 & 472 & 59 & 56 \\
\hline 4 & Paediatrics & 25 & 46 & 12 & 13 \\
\hline 5 & ENT & 2 & 4 & 0 & 2 \\
\hline 6 & ICCU & 23 & 96 & 07 & 16 \\
\hline 7 & Orthopaedics & 1 & 2 & 0 & 1 \\
\hline \multirow[t]{2}{*}{8} & Burn ward & 4 & 8 & 0 & 4 \\
\hline & Total & 267 & 1079 & 125 & 142 \\
\hline
\end{tabular}

TABLE 4:Indication of FFP use

\begin{tabular}{|l|l|l|l|}
\hline Sr no & Disease & No. of bags used & Percentage(\%) \\
\hline 1 & DIC & 389 & 36.05 \\
\hline 2 & Chronic liver disease & 185 & 17.14 \\
\hline 3 & Coagulation factor deficiency & 25 & 2.31 \\
\hline 4 & Hypovolemic replacement & 380 & 35.21 \\
\hline 5 & Excessive bleeding & 42 & 3.89 \\
\hline 6 & Surgical bleeding & 32 & 2.96 \\
\hline 7 & Rodenticide poisoning & 12 & 1.11 \\
\hline 8 & Snake bite & 14 & 1.29 \\
\hline
\end{tabular}

\section{Conclusion}

After evaluating the usage of FFP, it was found that there is a generalized and widespread irrational use of FFP among specialists. To reduce the inappropriate usage of FFP the following strategies may be used

1.The hospital transfusion guidelines should be established based on existing internatioinal guidelines.

2.Awareness program for the clinician should be conducted regularly.

3.In the requisition forms the appropriate indication for FFP transfusion should be mentioned regularly.

\section{References}

[1]. Nagareka kulkarni , Evaluation of fresh frozen plasma usage at a medical college hospital-A two year study, International journal of blood transfusion and immunohematology,vol 2,2012,16-20.

[2]. Meltem AKKAS, Denya KOMUR ATAMAN, Canan AKMAN , Nalan METIN AKSU, Mehmet Ali KARACA , Salih AKSU , Mehmet Mahir OZMEN , Inappropriate fresh frozem plasma use in coagulation disorder, European journal of surgical science , 2011;2(2):38-41.

[3]. medicine, international 2012,2(1);19-24.

[4]. Practice parameter for the use of FFP, cryprecipitate and platelets,.Administration practice guidelibnes, development task force of the cologe of american pathologist, JAMA 1994; 271(10); 777-81

[5]. National health and medical research council, clinical practice guidelines on the use of blood components 2001. 